\title{
OBJETOS DE REALIDAD AUMENTADA: PERCEPCIONES DEL ALUMNADO DE PEDAGOGÍA
}

\section{OBJECTS OF AUGMENTED REALITY: PERCEPTIONS OF PEDAGOGY STUDENTS}

\author{
Dra. Sandra Martínez Pérez ${ }^{1}$ \\ smartinezperez@ub.edu \\ Dra. Bárbara Fernández Robles ${ }^{2}$ \\ bfernandezrobles@gmail.com \\ ${ }^{1}$ Universidad de Barcelona, Facultad de Educación, Depto. Didáctica y Organización \\ Educativa, Campus Mundet, Pg. de la Vall d'Hebron, 171, Ed. Llevant, $2^{a}$ Planta, \\ Barcelona, 08035. \\ ${ }^{2}$ Universidad Isabel I, Facultad de Humanidades y Ciencias Sociales, Sede central \\ Burgos, C/ Fernán González, 76, Burgos
}

Resumen: La Realidad Aumentada (RA) se presenta como una tecnología emergente que ofrece grandes posibilidades a la sociedad en general, y a la formación universitaria en particular, debido a las oportunidades que presenta para crear entornos de aprendizaje atractivos, activos y constructivistas. La investigación que presentamos perseguía conocer la percepción que tienen los alumnos del Grado de Pedagogía sobre las potencialidades y dificultades de esta tecnología en educación, y empoderar al alumnado para que diseñaran contenidos en formato de objetos de RA. Para recabar la información se diseñó un cuestionario compuesto por 23 items en escala Likert. La muestra del estudio estuvo formada por 78 estudiantes del $2^{\circ}$ curso del Grado de Pedagogía de la Universidad de Sevilla, que cursaban la asignatura Tecnología Educativa. Como resultados de esta investigación, podemos destacar las percepciones positivas que tienen los futuros profesionales de la educación sobre esta tecnología y, especialmente los beneficios que aporta el uso de la misma en los procesos de aprendizaje.

Palabras clave: Realidad Aumentada, Pedagogía, encuesta, estudiantes universitarios Abstract: Augmented Reality (AR) is presented as an emerging technology that offers great possibilities to society in general, and to university education in particular, due to the opportunities it presents to create learning environments, active and constructivist. The research that we present will seek to know the perception that the students of the Degree of Pedagogy have about the potentialities and difficulties of this technology in education, and empowers the student to design the contents of the RA. To collect the information, a questionnaire was designed consisting of 23 items on a Likert scale. The sample of the study was formed by 78 students of the 2nd year of the Degree of Pedagogy of the University of Seville, who were taking the course Educational Technology. As results of this research, we can highlight the positive perceptions that future professionals of education have about this technology and, especially, the benefits that the use of it provides in the learning processes. Keywords: Augmented Reality, Pedagogy, survey, university students

Píxel-Bit. Revista de Medios y Educación. No 53 Julio 2018. ISSN: 1133-8482. e-ISSN: 2171-7966. doi: http://dx.doi.org/10.12795/pixelbit.2018.i53.14 


\section{Introducción}

A pesar de los cambios frenéticos de las tecnologías, la educación marca su propio ritmo, y es por ello que surge la necesidad de hablar de aplicaciones de las tecnologías que nos lleva a hablar y reflexionar en torno a los modelos flexibles de la enseñanza, las prácticas educativas y los entornos de aprendizaje (Castañeda, et al., 2016). Las diversas tecnologías emergentes (gamificación, MOOC, internet de las cosas, entornos personales de aprendizaje, web semántica, etc.) han calado de manera directa en el mundo educativo gracias a Internet, a la web 2.0 y a los dispositivos móviles. Un ejemplo de esas tecnologías emergentes es la Realidad Aumentada (RA) que está alcanzando un gran impacto en las instituciones educativas. Tal y como señalan Cabero y Barroso (2016a: 102) los escenarios de RA «favorecen el que los alumnos puedan contextualizar la información, y al mismo tiempo enriquecerla con información adicional en diferentes soportes y sistemas simbólicos». Así pues, desde esa óptica, dicha tecnología permite que el estudiantado pueda diseñar, crear e interactuar con objetos virtuales con el propósito de alcanzar una actitud positiva y un alto grado de satisfacción a partir de la puesta en marcha de la misma (Wojciechowski y Cellary, 2013).

Implementar la RA en los contextos educativos universitarios significa dar respuesta a las necesidades que se plantean en el día a día y mejorar las acciones formativas de la comunidad educativa. Con este propósito, nace el proyecto «Realidad Aumentada para Aumentar la Formación. Diseño, Producción y Evaluación de Programas de Realidad Aumentada para la Formación Universitaria (RAFODIUN)
(EDU2014-57446-P), con el fin de analizar las posibilidades educativas que para contextos de formación universitaria puede tener la RA, desde una doble mirada: tanto tecnológicasinstrumentales, como educativas, de diseño de entornos formativos, hasta la referida a la formación y el perfeccionamiento del profesorado, y al alumnado como productor de contenidos en RA. Y en concreto, el presente estudio tiene como foco central la Facultad de Ciencias de la Educación de la Universidad de Sevilla, concretamente la asignatura de Tecnología Educativa del Grado de Pedagogía.

\subsection{Tecnologías emergentes}

Las tecnologías de la información y la comunicación (TIC), especialmente la red Internet en todas sus aplicaciones, además de las redes sociales y todos los recursos llamados «web 2.0», están abriendo nuevas posibilidades para la ciudadanía (se habla, de forma un poco rimbombante, y más como deseo que como realidad, de la democracia electrónica), con unas nuevas formas de participación. (Aguaded y Cabero, 2014: 69).

La presente era digital está dando paso a novedosas maneras de interactuar con la realidad mediante las «nuevas» tecnologías interactivas y emergentes, aproximando a la ciudadanía a una cultura del conocimiento colaborativo (Cope y Kalantzis, 2010, Aguaded y Cabero, 2014), cuyas tendencias tienden a la privacidad, al contenido conectado, la globalidad como proceso, el uso del móvil, una identidad digital y el b-learning, entre otras. Estudiantado y profesorado nos vemos inmersos en desarrollar ciertas habilidades y realizar multitareas al mismo tiempo y, a su vez, a generar un aprendizaje ubicuo, conectado y fugaz. Las nuevas 
tecnologías están representando unas tendencias de vanguardia en educación, mientras algunas se están implementando otras están en el horizonte más próximo (DeLoatch, 2015). En esta sociedad digital líquida, estas tecnologías emergentes se presentan por un lado como aquellas herramientas informáticas clave que además de utilizarse hoy también prometen un crecimiento importante en los próximos años (Sharples, et al., 2014; Johnson, et al., 2015); $\mathrm{y}$ por otro, como un nuevo entramado tecnológico que desdibujan y presentan nuevas escenografías y proporcionan otras maneras de comunicarnos, compartida y abiertamente, tanto de manera sincrónicas como asincrónicas, generando nuevos entornos ricos de aprendizaje y dando paso a diferentes lugares (Fernández, 2017). En esta línea, Cabero y Barroso (2016) identifican como tecnologías emergentes: la gamificación, la Realidad Aumentada (en la que centraremos la mirada en este artículo), los MOOCs, la web semántica, los entornos de aprendizaje, el Internet de las cosas, flipped classroom y el Sistema de Gestión del Aprendizaje (LMS).

Si centramos la mirada en el ámbito educativo, las tecnologías emergentes según Marín (2017) presentan una serie de ventajas y beneficios, como por ejemplo, la motivación e intereses por parte del estudiantado en su proceso de aprendizaje, estimula la percepción, permite interactuar con la realidad en tiempo real, mejora el aprendizaje y la adquisición del conocimiento de una manera lúdica y mejora habilidades espaciotemporales y cognitivas.

\subsection{La Realidad Aumentada en Educación}

La incorporación de tecnologías portátiles digitales en nuestra vida diaria está dando lugar a nuevas metodologías de aprendizaje, incidiendo de forma directa en la manera de transmitir el conocimiento y de organizar la formación (Fombona y Vázquez-Cano, 2017). Estos cambios hacen que sea imprescindible conocer de cerca las tendencias en educación, con el fin de afrontar con actitud, compromiso y responsabilidad las demandas de la sociedad (Martínez y Torres, 2017).

En este sentido, son diversos los autores que han manifestado el impacto que está teniendo la realidad aumentada en este sector en los últimos años (Duh y Klopher, 2013; Cabero y Barroso, 2016b; Fombona y Vázquez-Cano, 2017), señalando que su uso puede provocar grandes beneficios al proceso de enseñanza-aprendizaje.

La realidad aumentada permite interaccionar con objetos reales y virtuales en tiempo real, dejándonos apreciar el objeto desde diferentes perspectivas y bajo diferentes soportes, a la vez que se enriquece la información existente (El Sayed, Zayed y Sharawy, 2011; Bower et al., 2013). Estas características interactivas facilitan la explicación de temas abstractos y de difícil acceso, evitando riesgos físicos que en muchas ocasiones se pueden producir al trabajar ciertos temas, como pueden ser los relacionados con laboratorios (Cabero y Barroso, 2016b).

El uso de RA ofrece la posibilidad de enriquecer libros y apuntes, de esta forma se reduce el material impreso y se enriquece con diversos recursos interactivos. Esta relevancia se refleja en el proyecto RAFODIUN o en el libro interactivo de monumentos andaluces (Ruiz, 2011), donde 
emplean la realidad aumentada como medio para mejorar la información existente con materiales digitales.

Además, su utilización puede ser un gran aliado para innovar en los procesos formativos y mejorar las metodologías de aprendizaje, dando respuesta a los diferentes estilos de aprendizaje y lugares de estudio del alumnado (Fombona y Vázquez, 2017). En este sentido, no podemos negar las grandes posibilidades que nos ofrece para trabajar con metodologías activas y constructivistas (Duh y Klopher, 2013), donde los alumnos puedan llevar su propio ritmo de aprendizaje (Wojciechowski y Cellary, 2013; Del Cerro y Morales, 2017).

Tal y como señalan Bower et al. (2013) su uso puede reducir la carga cognitiva al presentar un andamiaje situado. Igualmente, Cheng (2017) hace alusión a este tema, destacando al mismo tiempo que su uso fomenta actitudes positivas hacia el aprendizaje.

De igual modo y de acuerdo con Marín (2017), esta tecnología ofrece grandes oportunidades para trabajar en entornos inclusivos. Hecho que han demostrado Chen, Lee y Lin (2016) en una investigación puesta en marcha con niños autistas, en la que manifiestan que el uso de realidad aumentada influye de forma positiva en las habilidades sociales y en la atención de personas con Trastorno de Espectro Autista.

Por otro lado, son diversos los autores que han puesto de relieve que el uso de realidad aumentada incide de forma directa en la motivación de los alumnos (Di Serio, Ibáñez y Delgado, 2013; Redondo et al., 2014; Del Cerro y Morales, 2017). Este es el caso de Redondo et al. (2014), quienes demuestran en un estudio llevado a cabo con estudiantes universitarios de arquitectura que esta herramienta influye de forma positiva tanto en la motivación como en el rendimiento de los discentes. De igual modo, Del Cerro y Morales (2017) hacen alusión a la influencia que tiene el uso de recursos de realidad aumentada en la motivación de los estudiantes de secundaria, haciendo hincapié en que determina tanto la motivación como los resultados de aprendizaje.

Pero a pesar de los grandes beneficios que nos ofrece esta tecnología en la enseñanza, no podemos perder de vista que la tecnología en si no produce cambios, por lo que es necesario reflexionar antes sobre su utilización. En este sentido, Adell y Castañeda (2012) consideran que hay que ser prudentes ante el cambio revolucionario prometido por las tecnologías y que hay que mantener «un cierto grado de escepticismo» (p. 31). En esta línea, Fernández (2017) llama la atención a la importancia que tiene olvidar el efecto novedoso de la tecnología y en pensar más en su implementación y uso adecuado.

Para finalizar, no nos gustaría dejar atrás que nos encontramos con grandes desafíos para su incorporación en la enseñanza, que van desde la falta de buenas prácticas que sirvan de ejemplo y de estudios que se centren en su uso didáctico hasta la falta de formación del docente.

Por lo comentado, es relevante la realización de proyectos e investigaciones que sirvan para promover el uso adecuado de realidad aumentada, y que sean un apoyo para el desarrollo de buenas prácticas.

Con este objetivo, surge el proyecto RAFODIUN, el cual tiene entre otros fines promover la utilización educativa de esta herramienta tecnológica. 


\subsection{RAFODIUN: Un proyecto para la formación}

El proyecto $\mathrm{I}+\mathrm{D}+\mathrm{i} \ll$ Realidad aumentada para aumentar la formación. Diseño, producción y evaluación de programas de realidad aumentada para la formación universitaria» (EDU-5746-P - Proyecto RAFODIUN), financiado por el Ministerio de Economía y Competitividad del Gobierno de España, persigue varios objetivos, que van desde «Evaluar las posibilidades y potencialidades que ofrecen diferentes softwares utilizados para la creación de entornos tecnológicos bajo la arquitectura de la RA» hasta «Conocer las posibilidades educativas que permite que el alumno se convierta en productor de experiencias formativas apoyadas en RA».

Igualmente, entre sus fines encontramos diseñar y producir distintos contenidos en formato RA para ser aplicados en contextos de formación universitaria en distintas áreas curriculares, y evaluar sus posibilidades de cara al rendimiento de los alumnos.

En relación a este último objetivo, encontramos que son diversos los recursos de realidad aumentada que se han desarrollado para incorporarse en procesos formativos de diferentes áreas curriculares, los cuales pueden observarse en la página Web del Proyecto (http://intra.sav.us.es/ proyectorafodiun/index.php/objetos-en-ra).

En este sentido, podemos ver como se han diseñado objetos de RA de diferente índole, desde recursos de RA que tratan sobre anatomía hasta objetos de RA que se centran en temas educativos.

Dichos recursos han sido creados en el Secretariado de Recursos Audiovisuales de la Universidad de Sevilla (SAV), que tiene como fin promover el uso de las TIC en la educación universitaria.

Con el objetivo de dar respuesta a las demandas de la comunidad universitaria en lo que a realidad aumentada se refiere, este centro ha creado una convocatoria de Producción de Recursos de RA para diferentes áreas académicas de la Universidad de Sevilla. Dichos objetos de RA se pueden visualizar en la página Web del proyecto RAUS (http://realidadaumentada.us.es/).

Además, comentar que dentro de este Proyecto se ha creado la comunidad virtual RAFODIUN (Google+), con la que se persigue intercambiar noticias de interés sobre realidad aumentada. De esta forma, los diferentes miembros de la comunidad comparten noticias, artículos y aplicaciones que pueden ser de interés para el contexto educativo.

Lo comentado, resulta de gran utilidad, puesto que promueve el conocimiento de esta tecnología a la vez que facilita su uso adecuado en educación.

Por otro lado, como ya hemos resaltado, dentro del proyecto RAFODIUN se están desarrollando diversas experiencias con realidad aumentada en diversas áreas curriculares de nivel universitario. Entre estas podemos destacar desde las que se han desarrollado en el área de medicina (Barroso, Cabero y Moreno; 2015) hasta las desarrolladas con futuros profesionales de la educación (Barroso y Gallego, 2017; Fernández, 2017).

Comentar que la investigación que presentamos en el presente artículo también se ha puesto en marcha dentro de este proyecto, concretamente se ha desarrollado en el Grado de Pedagogía de la Universidad de Sevilla. Seguidamente, presentamos más detalles sobre la misma. 


\section{Metodología}

La presente investigación se llevó a cabo en el último trimestre del 2017. El objetivo general era analizar las posibilidades educativas que ofrece la Realidad Aumentada en los contextos de formación universitaria. Para ello fue necesario definir y reflexionar sobre el concepto de RA, empoderar al alumnado para que diseñaran contenidos en formato de objetos en RA para ser aplicados en contextos de formación universitaria y valorar y analizar las percepciones del estudiantado sobre los usos, potencialidades y dificultades de la misma.

\subsection{Participantes}

La muestra estuvo compuesta por 78 estudiantes del $2^{\circ}$ curso de la asignatura de Tecnología Educativa del Grado de Pedagogía de la Universidad de Sevilla, siendo el 92.3\% mujeres y 7.7\% hombres. En relación a los grupos, el 56.4\% del alumnado corresponde al de la mañana y el $43.6 \%$ al de la tarde.

\subsection{Instrumentos}

Para poder recoger el grado de satisfacción por parte del alumnado en relación al uso de la Realidad Aumentada, elaboramos un cuestionario (ver tabla 1) con 23 ítems en escala Likert 1 (totalmente en desacuerdo) 4 (totalmente de acuerdo). El diseño de las preguntas vino marcado por las necesidades que presentaban el alumnado y las condiciones del presente estudio. Es por ello que se procedió a la adaptación de dos instrumentos: a) el TAM (Modelo de Aceptación de las Tecnologías) y b) la UTAUT (Teoría Unificada de Aceptación y Utilización de la Tecnología). El TAM (Davis, 1989) tiene como finalidad conocer la utilidad y la fiabilidad de uso que el estudiantado percibe sobre la tecnología aplicada, teniendo en cuenta la influencia social y los procesos cognitivos como determinantes de este modelo (Venkatesh y Davis, 2000). Este modelo, también nos indica según (Venkatesh y Bala, 2008): la capacidad que la persona tiene para trabajar con la tecnología, la percepción del control externo, disfrute del uso de la tecnología, la usabilidad objetiva, el miedo que provoca el uso de la misma y la satisfacción - alegría del uso. La UTAUT (Venkatesh et al, 2003) no sólo tiene presente las expectativas del resultado y del esfuerzo y la influencia social; sino también las variables individuales (edad, sexo, experiencia y voluntariedad) en la intención y uso de la «innovación». Esta teoría, tal y como manifiestan y han adaptado los autores Briz, Méndez y García (2016), pretende conocer la valoración de aceptación del uso de la «nueva» tecnología móvil, en nuestro caso la puesta en marcha y la creación de objetos en Realidad Aumentada por parte del estudiantado.

\section{Resultados}

Los resultados obtenidos ante la usabilidad y la puesta en marcha de la «nueva» tecnología emergente, la Realidad Aumentada, nos revelan que el estudiantado ha utilizado en su mayoría el Smartphone (88.5\%) como herramienta para descargarse las Apps necesarias para el diseño y elaboración de sus objetos. Sólo un 1.3\% afirma haber utilizado la tableta para la descarga de las mismas y el resto reconoce que han manejado ambos dispositivos, el Smartphone y la tableta para la descarga de la aplicación. 


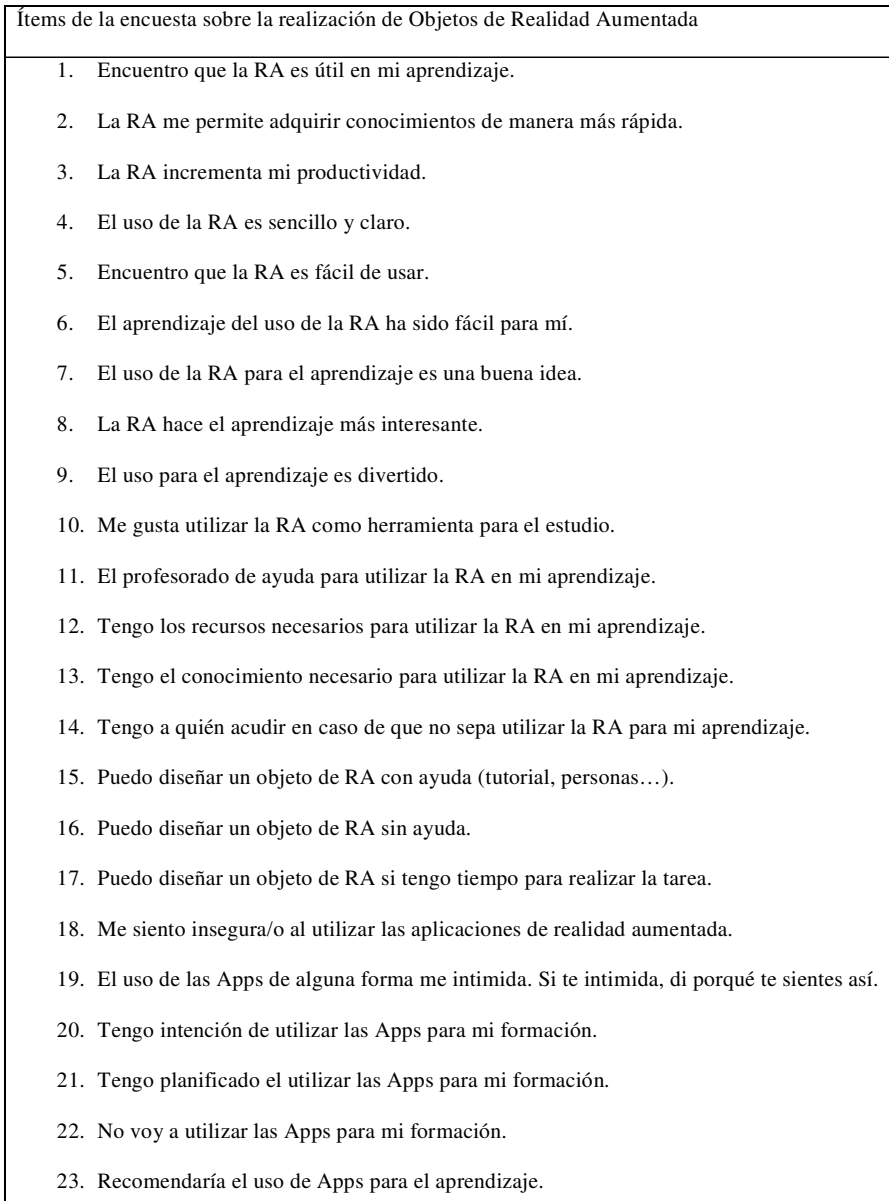

\section{Tabla 1: Cuestionario sobre la realización de Objetos de Realidad Aumentada}

Con respecto a la primera pregunta: Encuentro que la RA es útil para mi aprendizaje, tal y como se observa en la gráfica 1, la mayoría del estudiantado la consideran como una herramienta valiosa y beneficiosa para su proceso de aprendizaje, sólo un $10.3 \%$ no la perciben como tal:

En relación a los conocimientos adquiridos, según nos indica la gráfica 2 , un $79.5 \%$ del alumnado considera que la Realidad
Aumentada puede y es una tecnología que ayuda a la adquisición de nuevos conocimientos.

Con respecto a la productividad, en la gráfica 3, observamos que el $35.9 \%$ opinan que no incrementan la productividad en su aprendizaje, siendo, por lo contrario, un 64.1\% quiénes la vislumbran como posibilidad de crecimiento del rendimiento dentro del propio proceso de aprendizaje. 


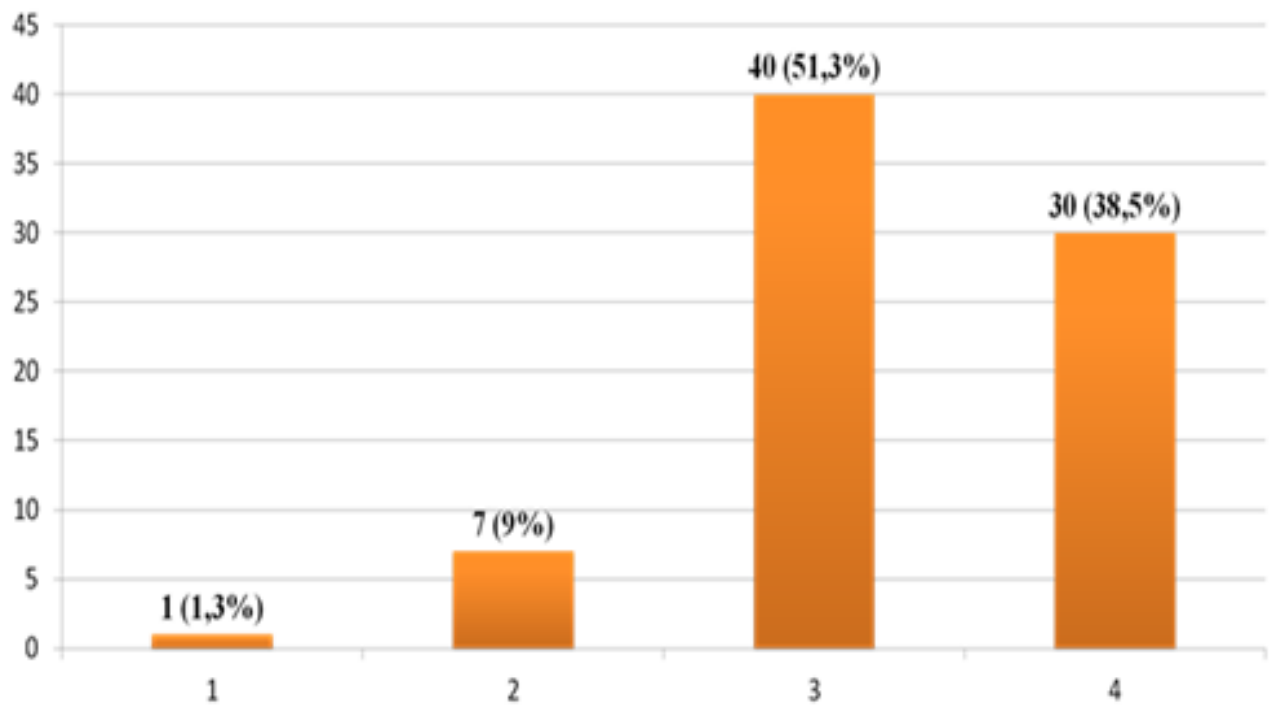

Gráfica 1: Utilidad de la RA para el aprendizaje

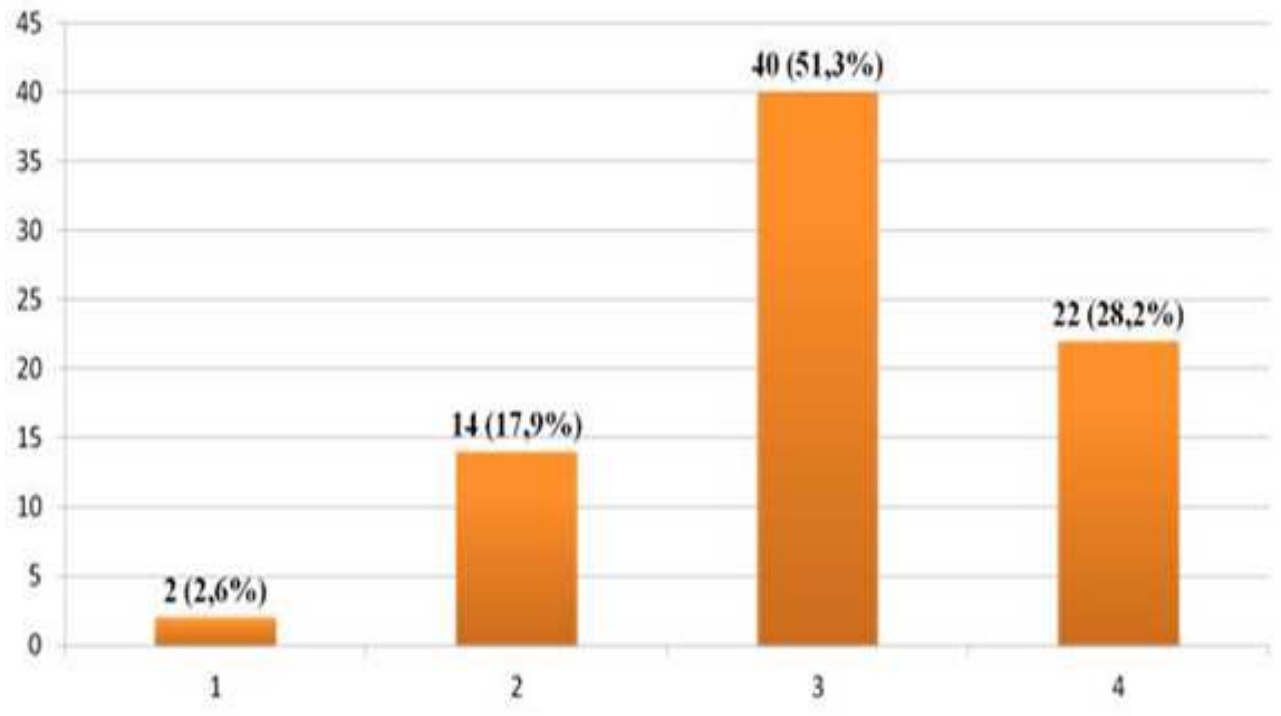

Gráfica 2: Adquisición de conocimientos

En cuanto a la sencillez y facilidad en el uso, el $48.7 \%$ están de acuerdo y totalmente 


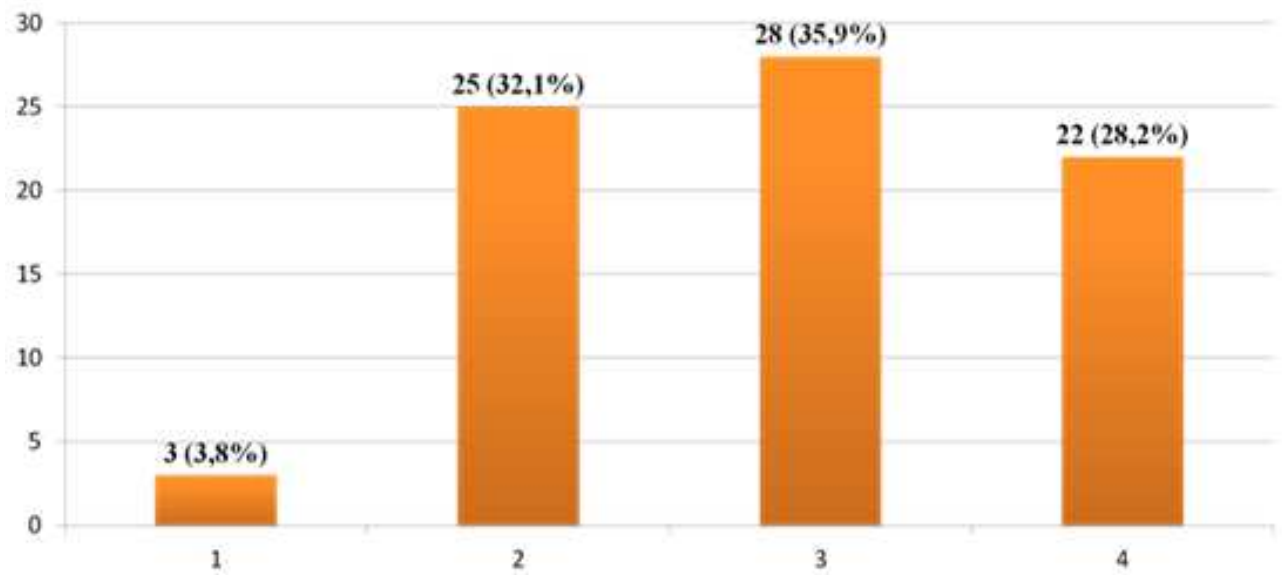

Gráfica 3: Incremento de la productividad

de acuerdo en cuanto la funcionalidad de la misma. Por el contrario, un 51,3\% tienen la percepción de que el uso de la nueva tecnología no es sencillo ni fácil de usar. Tal y como se observa en la gráfica 4 , el $46.2 \%$ identifican y están de acuerdo que el aprendizaje del uso de la RA ha sido relativamente fácil, mientras que $12.8 \%$ están en total desacuerdo de la facilidad de la misma.
Junto a esta percepción, el $44.9 \%$ y el $37.2 \%$ están de acuerdo y totalmente de acuerdo con el uso de la Realidad Aumentada para el aprendizaje, valorándola como una buena idea la aplicabilidad de la misma. Además, se ha señalado a su vez lo interesante y divertido que es para el aprendizaje con un total del $83.4 \%$.

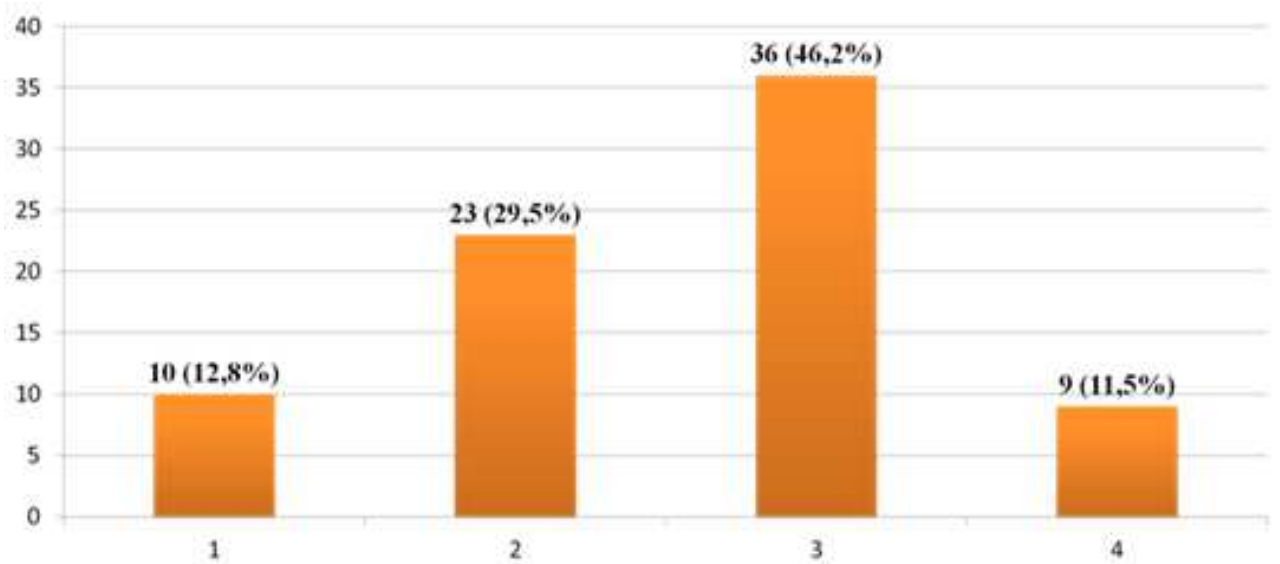

Gráfica 4: Facilidad del uso de la RA 
Una visión más positiva, la relacionamos el rol que desempeña el profesorado en el proceso de aprendizaje. El 76.9\% del estudiantado vislumbra la figura docente como ayuda y guía para utilizar la RA y tienen en cuenta que pueden acudir a él en el caso de que no se sepa utilizar para el propio proceso de aprendizaje. De esta manera, el $78.2 \%$ consideran tener los recursos necesarios para utilizar dicha tecnología y, a su vez, el 75.7\% manifiestan obtener los conocimientos necesarios para poder aplicar la RA en su entorno. La percepción que tiene el alumnado de todo ello es que el $92.3 \%$ pueden diseñar Objetos de RA con ayuda no sólo de personas, sino también mediante tutoriales elaborados para tal fin. Y el 84.6\% reconocen que son capaces de diseñarlos siempre y cuando dispongan del tiempo suficiente para ello.

Por otro lado, encontramos la visión menos positiva de la utilización de la herramienta. El $53.9 \%$ se siente insegura a la hora de utilizar aplicaciones en Realidad Aumentada. Un total del $83 \%$ nos comentan que el uso de este tipo de App le intimida por varias razones: por problemas con la aplicación (da fallos), por «miedo» a que no salga aquella idea que se pretende crear, por ser una app nueva y no dominarla correctamente, por incertidumbre, por no entenderla y tener conocimiento, por falta de competencias y práctica o, incluso, porque no le inspira confianza a la hora de guardar por si se pierde el trabajo realizado.

Por último, en el plano más de formación, el $60.3 \%$ tienen intención de seguir utilizando las apps de RA, el 51.2\% la contempla en su planificación como uso en su proceso formativo y, el 53.8\% están totalmente en desacuerdo que no la vayan a utilizar para su formación.
Así pues, se observa que el 79.5\% recomienda el uso de esta tecnología y sus apps para crecimiento y gestión de su propio proceso de aprendizaje.

\section{Discusión y conclusiones}

A lo largo de esta investigación hemos vislumbrado que el rol del profesor en esta sociedad ubicua «no es sólo un pedagogo, sino un planificador, un diseñador y un director» (Burbules, 2012, p.13), es un guía y acompañante. Y el del estudiantado ser diseñadores y productores de objetos de Realidad Aumentada, desarrollando, de esta manera, habilidades para el autoaprendizaje, trabajando en equipo y en red, siendo responsables de la búsqueda del contenido y de la información.

El docente influye y facilita el uso de RA, hecho que es demostrado cuando los alumnos ponen de manifiesto que ha ejercido un gran papel para facilitar el uso de RA, resultados similares a los obtenidos por Fernández (2017), quien afirma que los alumnos apreciaban la realidad aumentada fácil de usar gracias a las explicaciones realizadas por el profesor y a las guías facilitadas sobre su uso.

Por lo tanto, podemos decir que el uso de RA debe ir acompañada de una guía sobre su utilización y que el proceso debe ser coordinado por una persona (De la Horra, 2017).

Hemos observado a partir de las percepciones del estudiantado que la incorporación de la misma en los procesos de enseñanza-aprendizaje, ayuda a fines didácticos y pedagógicos, fomenta el uso y posibilidades de las tecnologías, promueve la motivación, la curiosidad y la creatividad, potenciando, a su vez, las prácticas 
pedagógicas y dibujando escenarios educativos enriquecedores. Escenarios que requieren una mínima formación digital por parte del alumnado, por la falta de conocimientos y habilidades al ser una tecnología emergente y, además, modelos flexibles de enseñanza.

Lo comentado, demuestra que los futuros profesionales de la educación aprecian los grandes beneficios que puede aportar su uso para enriquecer y facilitar las acciones formativas, resultados que están en consonancia con los adquiridos por otros autores que han utilizado RA en procesos formativos de futuros profesores (Cózar et al., 2015; Barroso y Gallego, 2017; Fernández, 2017).

Además, los estudiantes señalan que su utilización es interesante y divertido, aspecto que es importante, puesto que como han demostrado diferentes autores (Teo y Noyes, 2011; Fernández, 2017) el disfrute percibido por parte de los docentes en formación es un factor que determina la intención de utilizar la tecnología.

De esta manera y a modo de cierre, teniendo en cuenta las valoraciones a partir de la aplicación de la encuesta, podemos afirmar las posibilidades que otorga la RA en los procesos de aprendizaje, fomentando la creatividad, el trabajo colaborativo y en equipo, la comunicación entre el estudiantado (Barroso y Cabero, 2015; Marín, 2017) y, sobre todo, se puede considerar como una tecnología que realza la mejora del aprendizaje en los contextos de la educación superior.

\section{Fuentes de financiación}

El presente trabajo ha sido desarrollado en el marco del proyecto de investigación I+D «Realidad Aumentada para Aumentar la
Formación. Diseño, Producción y Evaluación de Programas de Realidad Aumentada para la Formación Universitaria»(RAFODIUN) (EDU2014-57446-P), financiado por el Ministerio de Economía y Competitividad.

\section{Referencias bibliográficas}

Adell, J. \& Castañeda, L. (2012). Tecnologías emergentes, ¿pedagogías emergentes? En J. Hernández, M. Pennesi, D. Sobrino \& A. Vázquez (Coords). Tendencias emergentes en educación con TIC. (pp.1863). Barcelona: Editorial espiral.

Aguaded, I. \& Cabero, J. (2014). Avances y retos en la promoción de la innovación didáctica con las tecnologías emergentes e interactivas. Educar, especial 30 aniversario, 67-83. Recuperado de: http:// dx.doi.org/10.5565/rev/educar.691

Barroso, J., \& Cabero, J. (2015). Realidad Aumentada: posibilidades educativas. En Ruiz-Palmero, J., Sánchez-Rodríguez, J. y Sánchez-Rivas, E. (Edit.). Innovaciones con tecnologías emergentes. Málaga: Universidad de Málaga.E. (Edit.). Innovaciones con tecnologías emergentes. Málaga: Universidad de Málaga.

Barroso, J., Cabero, J. \& Moreno, A.M. (2016). La utilización de objetos de aprendizaje en realidad aumentada en la enseñanza de la medicina. Innoeduca. International Journal of Technology and Educational Innovation, Vol. 2. No. 2, 77-83.

Barroso, J. \& Gallego, O. (2017). Producción de recursos de aprendizaje apoyados en Realidad Aumentada por parte de los estudiantes de magisterio. Edmetic. Revista de Educación Mediática y TIC., 6, 1, 23-38.

Bower, M., Howe, C., McCredie, N., Robinson, A. \& Grover, D. (2014). Augmented 
Reality in education-cases, places and potentials. Educational Media International, 51 (1), 1-15. Recuperado de http:// www.tandfonline.com/toc/remi20/51/ 1 ?nav=tocList

Briz, L. J., Méndez, J. A. \& García, F. J. (2016). Recurso disponible de una encuesta basada en el modelo UTAUT para la aceptación de tecnologías móviles entre estudiantes y profesores. Salamanca: Grupo de Investigación GRIAL, Universidad de Salamanca, Disponible en http:// repositorio.grial.eu/handle/grial/600

Burbules, N. (2012). El aprendizaje ubicuo y el futuro de la enseñanza. Encuentros, 13, 3-14.

Cabero, J. \& Barroso, J (2016a). Posibilidades educativas de la realidad aumentada. En J. Cabero y F. García. Realidad Aumentada. Tecnología para la información, 97-112, Madrid: Editorial Síntesis.

Cabero, J. \& Barroso, J. (2016b). Ecosistema de aprendizaje con realidad aumentada: posibilidades educativas. CEF, 5, 141-154. Recuperado de http://tecnologia-cienciaeducacion.com/judima/index.php/TCE/ article/view/101

Castañeda, L., Prendes, Mª P. \& Gutiérrez, I. (2016). Pedagogías emergentes: tecnologías para la educación flexible. En J. Cabero y J. Barroso (Coords.) Nuevos retos en tecnología educativa. 195-216, Madrid: Editorial Síntesis.

Chen, C.H., Lee, I.J., \& Lin, L.Y. (2016). Augmented reality-based videomodeling storybook of nonverbal facial cues for children with autism spectrum disorder to improve their perceptions and judgments of facial expressions and emotions. Computers And Human Behaviour, 16, 477-485.

Cheng, K.H. (2017). Reading an augmented reality book: An exploration of learner's cognitive load, motivation and attitudes. Australasian Journal of Educational Technology, 33 (4), 53-69.

Cope, B. \& Kalantzis, M. (2010). Ubiquitous Learning. Chicago: University of Illinois Press.

Cózar, R., Del Valle, M., Hernández, J.A., \& Hernández, JR. (2015). Tecnologías emergentes para la enseñanza de las Ciencias Sociales. Una experiencia con el uso de Realidad Aumentada en la formación inicial de maestro. Digital education review, 27, 138153. Recuperado de http://revistes.ub.edu/ index.php/der/article/view/11622/pdf

Davis, F.D. (1989). Perceived usefulness, perceived ease of use, and user acceptance of information technology. MIS Quarterly 1 (3), 319-340. Recuperado de: http:// w w w.j s t o r.org/s t a b l e / 249008?seq=1\#page_scan_tab_contents

De la Horra, I. (2017). Realidad Aumentada, una revolución educativa. Revista Edmetic, 6 (1), 9-22. Recuperado de https:// www.uco.es/ucopress/ojs/index.php/ edmetic/article/view/5762

DeLoatch, P. (2015). 10 Emerging Education Technologies. Disponible en: http://www.edudemic.com/10-emergingeducation-technologies/

Del Cerro, F. \& Morales, G. (2017). Realidad Aumentada como herramienta de mejora de la inteligencia espacial en estudiantes de educación secundaria. RED. Revista de educación a distancia, 54, 1-14.

Di Serio, A., Ibáñez, M.B., \& Delgado, C. (2013). Impact of an augmented reality system on students' motivation for a visual art course. Computer \& education, 68, 586-596. Recuperado de http:// WwW.sciencedirect.com/science/article/pii/ S0360131512000590 
Duh, H.B.L., \& Klopher, E. (2013). Augmented reality learning: New learning paradigm in co-space. Computers \& education, 68, 534-535. Recuperado de http:/ /www.sciencedirect.com/science/article/pii/ S0360131513001978

El Sayed, N., Zayed, H.H., \& Sharawy., M. (2011). ARSC: Augmented reality student card.An augmented reality solution for the education field. Computers \& education, 56 (4), 1045-1061.Recuperado de http://0www.sciencedirect.com.fama.us.es/science/ article/pii/S0360131510003040

Fernández, B. (2017). La utilización de objetos de aprendizaje de realidad aumentada en la enseñanza universitaria de educación primaria. International Journal of Educational Research and Innovation (IJERI), 9, 90-104.

Fombona, J. \& Vázquez-Cano, E. (2017). Posibilidades de utilización de la geolocalización y realidad aumentada en el ámbito educativo. Educación XXI, 20 (2), 319342.

Johnson, L., Adams Becker, S., Estrada, V. \& Freeman, A. (2015). NMC Horizon Report: 2015 Higher Education Edition. Austin, Texas: The New Media Consortium.

Marín, V. (2017). The augmented reality in the educational sphere of student of degree in chilhood education. Case study. Pixel Bit: Revista de Medios y Educación 51, 7-19.nDoi: http://dx.doi.org/10.12795/pixelbit.2017.i51.01

Marín, V. (2017). The Relationships Between Augmented Reality and Inclusive Education in Higher Education. Bordon, 69 (3), 17-22.

Martínez, K., \& Torres, L.M. (2017). Estrategias que ayudan al docente universitario a conocer, apropiar e implementar las TIC en el aula. Mesa de innovación. Pixel Bit. Revista de medios y educación, 50,159-172.

Moreno, N.M., \& Leiva, J.L. (2017). Experiencias formativas de uso didáctico de la realidad aumentada con alumnado del grado de educación primaria en la universidad de Málaga. Revista Edmetic, 6 (1), 81-104. Recuperado de http://www.uco.es/ucopress/ ojs/index.php/edmetic/article/view/580

Redondo, E., Fonseca, D., Sánchez, A. \& Navarro, I. (2014). Mobile learning en el ámbito de la arquitectura y la edificación. Análisis de casos de estudio. Revista de Universidad y Sociedad del Conocimiento (RUSC) ,11 (1), 152-174. Recuperado de http:/ /www.raco.cat/index.php/RUSC/article/ viewFile/285042/373038

Ruiz, D. (2011). Realidad aumentada, educación y museos. Icono14, 9 (2), 212-226. Recuperado de http://icono14.net/ojs/ index.php/icono14/article/view/24

Sharples, M., Adams, A., Ferguson, R., Gaved, M., McAndrew, P., Rienties, B., Weller, M. \& Whitelock, D. (2014). Innovating Pedagogy 2014: Open University Innovation Report 3. Milton Keynes: The Open University

Teo, T., \& Noyes, J. (2011). An assessment of the influence of perceived enjoyment and attitude on the intention to use technology among pre-service teachers: A structural equation modeling approach. Computers \& education, 57 (2), 1645-1653. Recuperado de http://www.sciencedirect.com/science/ article/pii/S0360131511000637

Venkatesh, V. \& Davis, F.D. (2000). A theoretical extension of the technology acceptance model: Four longitudinal field studies. Management Science, 46 (2), 186204.

Venkatesh, V., Morris, M.G., Davis, G.B. \& Davis, F.D. (2003). User acceptance of 
information technology: Toward a unified view. MIS Quarterly, 27 (3), 425-478.

Venkatesh,V. \& Bala, H.(2008).Technology acceptance model 3 and a research agenda on interventions. Decision Sciences, 39 (2), 273-312. Recuperado de https://ai2-s2pdfs . s 3 . a m a z o n a w s . com/d 112 / d71f9dcd74cf1a44df50dee44bc48c6a9217.pdf

Wojciechowski, R. \& Cellary, W. (2013). Evaluation of learners' attitude toward learning in ARIES augmented reality environments. Computers \& Education, 68, 570-585.

Fecha de recepción: 29-01-2018 Fecha de evaluación: 13-02-2018 Fecha de aceptación: 26-02-2018 